\title{
COMMENT
}

\section{Evaluating anti-epileptic drugs in the era of therapeutic hypothermia}

\author{
Joanne O. Davidson ${ }^{1}$, Laura Bennet ${ }^{1}$ and Alistair J. Gunn (iD) ${ }^{1}$ \\ Pediatric Research (2019) 85:931-933; https://doi.org/10.1038/s41390-019-0319-6
}

In this issue, Soul et al. ${ }^{1}$ present recommendations for designing therapeutic trials for anti-epileptic drugs (AEDs) in neonates. This is an important resource. It is vital not to treat the neonate with seizures as a small adult. Rather, trials need to be appropriately designed for neonates and AEDs should be specifically developed and chosen for use in treating neonatal seizures. The authors highlight that neonatal seizures are highly heterogeneous. It is likely that the ideal therapeutic approach will be different for term and preterm infants, and for different forms of neonatal epilepsy (typically genetic or related to brain malformations), compared to the evolving seizures associated with acute injury such as neonatal encephalopathy (NE), stroke, or intracerebral hemorrhage, ${ }^{2}$ and so trials must be rigorously stratified by maturity and etiology. However, we respectfully disagree with the authors' suggestion that all clinical trials of AEDs should use phenobarbital as the control. Fully randomized controlled trials of AEDs versus placebo may be appropriate in the context of therapeutic hypothermia for infants with NE.

\section{NEONATAL SEIZURES}

Seizures are common in newborn infants who need neonatal intensive care. However, the consequences of seizure activity in the developing brain and the most effective way to treat these seizures remains surprisingly poorly understood. Although seizures in the neonate are largely regarded as a neurologic emergency requiring prompt treatment, in practice there is little evidence supporting a beneficial effect of AEDs and there is concerning evidence regarding potential side effects of these medications. Furthermore, it is not clear how the relative benefits and risks of AEDs have changed in the era of therapeutic hypothermia. Thus, optimal treatment of seizures remains controversial.

Seizures in the neonate appear to be different in many ways to those in the adult or even during childhood. The majority of neonatal seizures are a transient response to acute cerebral compromise, most commonly associated with NE. In infants with $\mathrm{NE}$, seizures usually start within the first days of life, their intensity reflects the severity of underlying cerebral injury and they tend to resolve over hours to days irrespective of treatment.

Biologically, there are multiple differences in neurotransmitter function during development that may increase the susceptibility of the immature brain to seizures compared to adults, and, at least potentially, may affect response to AEDs. Critically, there is a developmentally regulated switch from $\gamma$-aminobutyric acid stimulation of depolarization before birth, to hyperpolarization in the adult brain. This relative increase of excitation in the developing brain is important for development of neural circuitry, but likely increases susceptibility to seizures. ${ }^{3}$ Moreover, there is greater density of calcium-permeable, GluR2-subunit-deficient AMPA receptors, which contribute to a lower threshold for seizures, and expression of $\mathrm{N}$-methyl-D-aspartic acid (NMDA) glutamate receptor subunits (GluN) such as GluN2B, which promote prolonged excitatory post-synaptic potentials and GluN3A. ${ }^{4}$ Further, hypoxia can change the expression of NMDA receptor subunits, and so promote seizures in the developing brain. $^{5}$

The real-world impact of these biological differences is unknown, but they have important implications for the initiation and propagation of seizure activity as well as the efficacy and long-term neurodevelopmental effects of AEDs. Thus, it is vital to develop and test AED protocols specifically in neonates, and not rely on evidence from studies in adults.

\section{AEDS FOR THE TREATMENT OF NEONATAL SEIZURES}

The differences between the pathophysiology of adult and neonatal seizures are likely to contribute to the poor efficacy of current anti-seizure drugs in neonates. Consistent with older studies showing only partial efficacy of phenobarbital and phenytoin in treating neonatal seizures, in a recent prospective study of 611 preterm and term neonates presenting with seizures, $63 \%$ failed to respond to an initial loading dose of phenobarbital. ${ }^{6}$ The neonates most likely to respond to treatment are those with mild seizures or seizures that are already decreasing in severity before treatment. The evidence for a beneficial effect of antiseizure drugs in neonates is so limited that a 2007 Cochrane review concluded that "anticonvulsant therapy to term infants in the immediate period following perinatal asphyxia cannot be recommended for routine clinical practice, other than in the treatment of prolonged or frequent clinical seizures". ${ }^{7}$

Further, animal studies suggest that AEDs have the potential to cause unwanted side effects in the developing brain, including widespread apoptotic neurodegeneration and altered neuronal proliferation. For example, phenobarbital treatment was associated with a significant reduction in proliferation in the dentate gyrus and reduced expression of neuronal markers and neuronal transcription factors and neurotrophins in P4-P6 neonatal rats. ${ }^{8}$

Although it is difficult to determine whether AEDs have acute neurotoxic effects in human neonates, long-term exposure to phenobarbital for the treatment of febrile seizures was associated with a reduction in the development of language/verbal skills by

\footnotetext{
${ }^{1}$ The Department of Physiology, Faculty of Medical and Health Sciences, The University of Auckland, Auckland, New Zealand

Correspondence: Alistair J. Gunn (aj.gunn@auckland.ac.nz)
}

Received: 2 December 2018 Revised: 9 January 2019 Accepted: 21 January 2019

Published online: 30 January 2019 
school age with no difference in the recurrence of febrile seizures. ${ }^{9}$ In a secondary analysis of the National Institute of Child Health and Human Development randomized controlled trial of therapeutic hypothermia, severe $\mathrm{NE}$, anticonvulsant therapy, and mechanical ventilation were independently associated with 18month death/disability, whereas therapeutic hypothermia was protective. $^{10}$

\section{THERAPEUTIC HYPOTHERMIA AND SEIZURES}

Both clinical and preclinical studies support a beneficial effect of hypothermia on seizure activity in addition to its long-term neuroprotective effects. A study of 107 neonates with NE showed that although hypothermia did not reduce the number of patients with seizures, it was associated with a significant reduction in seizure burden in neonates with moderate, but not severe, NE. ${ }^{11}$ Further, a retrospective study of 56 neonates treated with therapeutic hypothermia for NE showed an association between hypothermia and reduced seizures within 6 months of discharge. ${ }^{12}$ Consistent with these findings, in a cohort study of 15 neonates with isolated focal ischemic stroke, $0 / 5$ children treated with hypothermia had seizures compared to $7 / 10$ who were not treated with hypothermia. ${ }^{13}$ It is important to appreciate that the effect of hypothermia on seizures likely reflects a combination of direct suppression of epileptiform activity plus indirect effects mediated through stabilizing cell survival. ${ }^{14}$ Regardless, this reduction in seizure number and intensity during therapeutic hypothermia has implications for clinical trials of AEDs in term and near-term infants.

Moreover, preclinical studies highlight the potential for AEDs to augment the beneficial effects of therapeutic hypothermia. A study in P7 rats exposed to hypoxia-ischemia $(\mathrm{HI})$, induced by single carotid artery ligation and inhalation hypoxia, showed that early administration of phenobarbital, $15 \mathrm{~min}$ after $\mathrm{HI}$, augmented neuroprotection with hypothermia started 1 or 3 hours after $\mathrm{HI}^{15}$ The same group have also shown that bumetanide in addition to phenobarbital and hypothermia augmented neuroprotection in the same model. ${ }^{16}$ Similarly, Krishna et al. ${ }^{17}$ also found augmentation of hypothermia with prophylactic phenobarbital in P10 rat pups subjected to unilateral HI. However, these studies are limited by the use of suboptimal durations of hypothermia (3-4h) that are not associated with benefit to sensorimotor or histological outcomes in these models. Further, AEDs were administered from either 10 or 15 min after $\mathrm{HI}$ in contrast with much more delayed use in clinical practice.

Clinically, a pilot evaluation in 14 neonates with HIE of bumetanide administered in conjunction with therapeutic hypothermia and phenobarbital was terminated early due to lack of prespecified efficacy and the finding that three infants developed hearing loss, leading to an unacceptable risk-tobenefit ratio. ${ }^{18}$ This study highlighted a number of important issues for future trials of AEDs in the era of therapeutic hypothermia. For example, the number of neonates who developed seizures when treated with therapeutic hypothermia was low and likely included a high proportion of neonates with relatively severe brain injury, who are less likely to benefit from treatment with either therapeutic hypothermia or phenobarbital.

A pertinent issue raised by Soul et al. ${ }^{1}$ is the ethical constraint around the use of placebos in clinical trials when there is a "standard" treatment, such as phenobarbital for seizures. However, given the poor efficacy of current AEDs for controlling neonatal seizures, the lack of evidence that they improve long-term outcome, concerns over adverse effects and uncertainty over their pharmacokinetics when administered in conjunction with therapeutic hypothermia, designing trials of new AEDs in the hypothermia era using an "add-on" method on top of phenobarbital is less than ideal and is likely to leave many important questions unanswered. Given that hypothermia is associated with reduced overall seizure burden, and has been shown to improve long-term outcomes and to be safe for use in neonates with $\mathrm{NE}$, at least in intensive care, it is reasonable to consider whether therapeutic hypothermia alone could be considered to be the standard treatment rather than phenobarbital, on top of which other AEDs could be randomized. This would allow researchers to establish which AED or combination of AEDs is most effective for use with therapeutic hypothermia in infants with NE. Is also important to include long-term follow-up in these studies, to determine whether the use of AEDs on top of therapeutic hypothermia is associated with any beneficial or detrimental longterm neurological effects.

It is essential to note that this approach could only be used for randomized controlled trials of AEDs in term and near-term neonates with NE who are treated with hypothermia. Nevertheless, such studies might suggest the most effective and safest $A E D(s)$ for further testing in term infants with seizures not associated with NE. Therapeutic hypothermia is not established in preterm infants with $\mathrm{NE}$, and the ideal $\mathrm{AED}$ is likely to be different again in preterm infants; they must not be considered to be "little term infants."

\section{VIDEO EEG AND SEIZURE DETECTION}

There is disagreement in the field about how to best detect neonatal seizure activity. We support the suggestion by Soul et al. ${ }^{1}$ that video EEG is essential to accurately detect seizure activity in neonates, in whom there is a high proportion of purely electrographic (subclinical) seizures. This is particularly important for detecting seizures in preterm neonates, who have been shown to have much higher rates of exclusively subclinical seizures compared to term neonates ( $24 \%$ vs. $14 \%){ }^{6}$ It is likely that total seizure burdens are underestimated because comprehensive monitoring is not available from early enough after birth.

\section{CONCLUSION}

Current AEDs are not ideal for use in neonates-they show limited efficacy, have high potential for adverse effects, and there is a striking lack of evidence that they improve long-term outcomes. Well-designed randomized controlled trials, stratified by etiology of seizures, are essential to develop evidence-based treatment strategies for the neonate with seizures.

\section{ACKNOWLEDGEMENTS}

This work was supported by the Health Research Council of New Zealand (17/601) and (16/003).

\section{AUTHOR CONTRIBUTIONS}

J.O.D., L.B., and A.J.G. contributed to the conception and design of the manuscript, drafting the article, and approving the final version.

\section{ADDITIONAL INFORMATION}

Competing interests: The authors declare no competing interests.

Publisher's note: Springer Nature remains neutral with regard to jurisdictional claims in published maps and institutional affiliations.

\section{REFERENCES}

1. Soul, J. et al. Recommendations for the design of therapeutic trials for neonatal seizures. Paediatr Res. (2018). https://doi.org/10.1038/s41390-018-0242-2.

2. Shellhaas, R. A. et al. Profile of neonatal epilepsies: characteristics of a prospective US cohort. Neurology 89, 893-899 (2017). 
3. Nardou, R., Ferrari, D. C. \& Ben-Ari, Y. Mechanisms and effects of seizures in the immature brain. Semin. Fetal Neonatal Med. 18, 175-184 (2013).

4. Jensen, F. E. Neonatal seizures: an update on mechanisms and management. Clin. Perinatol. 36, 881-900 (2009). vii.

5. Zhou, C., Sun, H., Klein, P. M. \& Jensen, F. E. Neonatal seizures alter NMDA glutamate receptor GluN2A and $3 \mathrm{~A}$ subunit expression and function in hippocampal CA1 neurons. Front. Cell. Neurosci. 9, 362 (2015).

6. Glass, H. C. et al. Seizures in preterm neonates: a multicenter observational cohort study. Pediatr. Neurol. 72, 19-24 (2017).

7. Evans, D. J., Levene, M. I. \& Tsakmakis, M. Anticonvulsants for preventing mortality and morbidity in full term newborns with perinatal asphyxia. Cochrane Database Syst. Rev. 2007. Art. No.: CD001240. https://doi.org/10.1002/ 14651858.CD001240.pub2.

8. Endesfelder, S. et al. Caffeine protects against anticonvulsant-induced impaired neurogenesis in the developing rat brain. Neurotox. Res. 34, 173-187 (2018).

9. Sulzbacher, S., Farwell, J. R., Temkin, N., Lu, A. S. \& Hirtz, D. G. Late cognitive effects of early treatment with phenobarbital. Clin. Pediatr. (Phila.) 38, 387-394 (1999).

10. Natarajan, G. et al. Association between sedation-analgesia and neurodevelopment outcomes in neonatal hypoxic-ischemic encephalopathy. J. Perinatol. 38, 1060-1067 (2018).
11. Low, E. et al. Cooling and seizure burden in term neonates: an observational study. Arch. Dis. Child. Fetal Neonatal Ed. 97, F267-F272 (2012).

12. Ghosh, S., Tran, L., Shuster, J. J. \& Zupanc, M. L. Therapeutic hypothermia for neonatal hypoxic ischemic encephalopathy is associated with short-term reduction of seizures after discharge from the neonatal intensive care unit. Childs Nerv. Syst. 33, 329-335 (2017).

13. Harbert, M. J. et al. Hypothermia is correlated with seizure absence in perinatal stroke. J. Child Neurol. 26, 1126-1130 (2011).

14. Wassink, G., Gunn, E. R., Drury, P. P., Bennet, L. \& Gunn, A. J. The mechanisms and treatment of asphyxial encephalopathy. Front. Neurosci. 8, 40 (2014).

15. Barks, J. D., Liu, Y. Q., Shangguan, Y. \& Silverstein, F. S. Phenobarbital augments hypothermic neuroprotection. Pediatr. Res. 67, 532-537 (2010).

16. Liu, Y., Shangguan, Y., Barks, J. D. \& Silverstein, F. S. Bumetanide augments the neuroprotective efficacy of phenobarbital plus hypothermia in a neonatal hypoxia-ischemia model. Pediatr. Res. 71, 559-565 (2012).

17. Krishna, S., Hutton, A., Aronowitz, E., Moore, H. \& Vannucci, S. J. The effects of adding prophylactic phenobarbital to therapeutic hypothermia in the termequivalent hypoxic-ischemic rat. Pediatr. Res. 83, 506-513 (2018).

18. Pressler, R. M. et al. Bumetanide for the treatment of seizures in newborn babies with hypoxic ischaemic encephalopathy (NEMO): an open-label, dose finding, and feasibility phase 1/2 trial. Lancet Neurol. 14, 469-477 (2015). 